\title{
DEFECTOSCOPIC SUSCEPTIBILITY OF MACHINE ELEMENTS
}

In the paper defectoscopic susceptibility of machine elements is analyzed. At first, an objective notion is defined and presented in a practical situation. The defectoscopic susceptibility as a feature can be considered and described also as a system consisting of two subsystems and a collection of non-technical factors. The subsystems are defined as a group of many factors. A mathematical structure of selected factors was shown as an example. Some factors of defectoscopic and diagnostic susceptibilities were calculated and gathered for selected parts of a motor vehicle and for air conditioning systems of selected models of cars. The calculated values of those factors enable us to quantitatively assess such features of a machine as its various susceptibilities. They can be practically used in the design, manufacture and maintenance not only of machines, but also of equipment for examination and service of machines and their subassemblies.

\section{Introduction}

The defectoscopic susceptibility is a feature which appears while carrying on nondestructive testing, and in particular, during defectoscopic examinations. It has been often indirectly mentioned in descriptions of nondestructive testing (ndt) method of examination. In some fields of technical activity, e.g. service, repair, diagnostic [1-5], these features are already considerably determined. The position of these features in the maintenance sphere of machines is shown in Fig. 1.

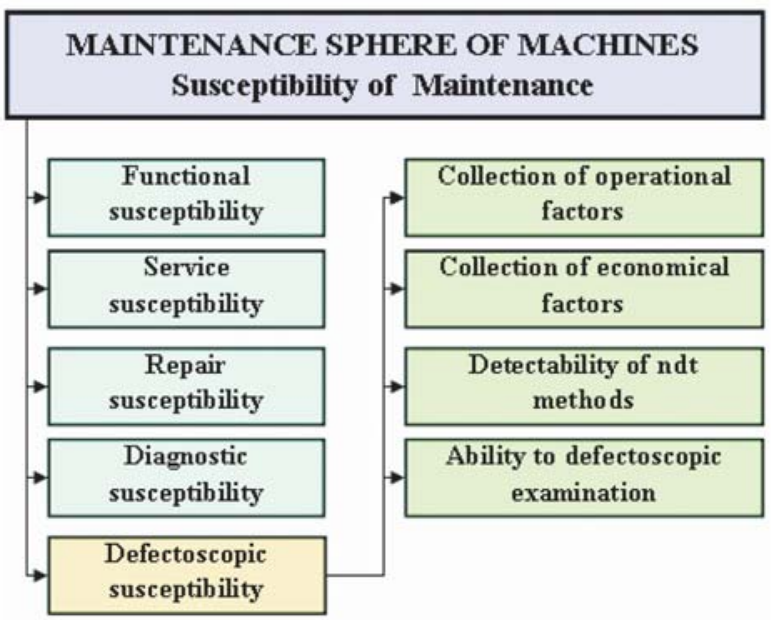

Fig. 1. The defectoscopic susceptibilities as components of maintenance susceptibility of machines

These features could be considered as a system consisting of a subsystem of the elements ability to defectoscopic examination and a subsystem of detectability of ndt methods to flaw detection.
These subsystems could be described by the collection of factors. The number of factors has not yet been exactly determined, but the most important of them are known and are included in the paper.

A process of the form of defectoscopic susceptibility occurs during all stages of existence of technical objects, and especially in their design and manufacturing. Both of the mentioned subsystems allow us to characterize the adaptation of the machine element to ndt evaluation and also the ability of means of control to flaw detection in quantitative category. Other susceptibilities characterizing the sphere of machine maintenance are defined as the object of action in this sphere (machines, their subassemblies, parts) as well as the means of action (i.e. methods and technological or measuring equipment) and are used for a given kind of technical activity.

\section{Presentation of the problem}

Each operator, which executes the defectoscopic control, could meet the problem analyzed in the paper. The problem occurs indirectly during control as a description of the control process. In such a description the defectoscopic susceptibility is, as a rule, characterized qualitatively.

In another field of technology and maintenance of machines, e.g. diagnostics and service, a parameterization of such features as diagnostic susceptibility and exploitational or service susceptibility of an object have been carried out. In the domain of defectoscopic control, quantitatively defectoscopic susceptibility is needed. The need has practical meaning. It enables us the so-called friendly defectoscopic examination [6].

The problem analyzed with the aid of simple practical examples of nondestructive ultrasonic control is illustrated in Fig. 2.

\footnotetext{
* Marian Josko

Poznan University of Technology, Institute of Working Machines and Motor Vehicles, Piotrowo 3, 60-965 Poznan, Poland,

E-mail: Marian.Josko@put.poznan.pl
} 
Adaptation of machine part

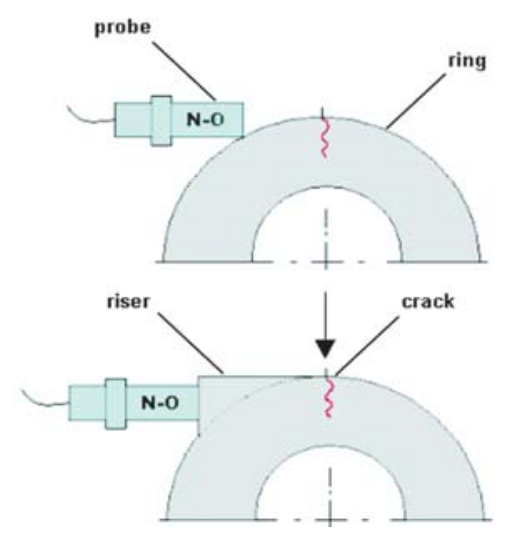

Adaptation of ultrasonic probe

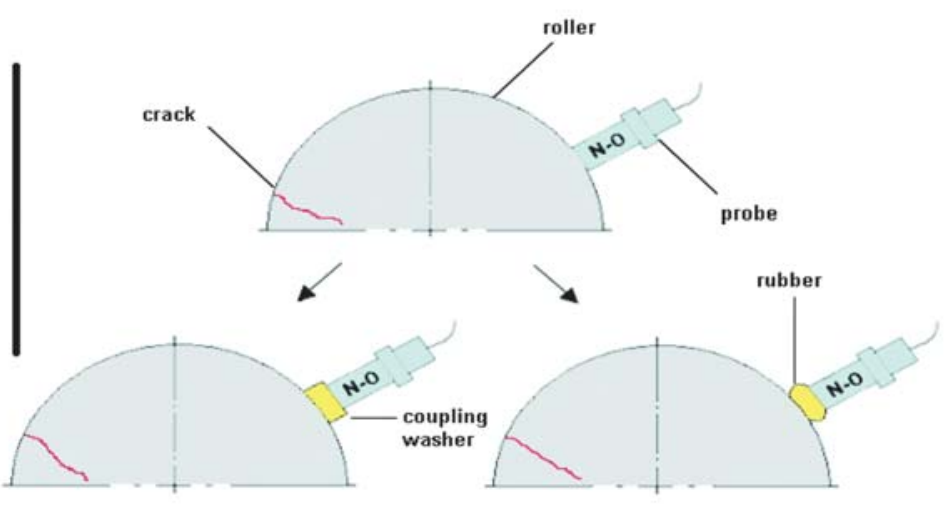

Fig. 2. An illustration of the presented problem in practical operating examples

A detection of discontinuity in parts of machine (ring or roller) by using a typical ultrasonic probe can be resolved in two ways. The first way is connected with designing and carrying out of a riser on ring, which makes ultrasonic monitoring possible in the critical area of the ring. This way is named as adaptation of the parts to ultrasonic examination. The second way is based on the use of coupling means between the probe and the roller. That way can be named as adaptation of the probe to ultrasonic examination. In this case, as a rule, possibilities of adaptation are greater than in the first case.

\section{The ability of elements to defectoscopic examination}

This feature describes an object of examination, e.g. parts of machines and their connections. Among many factors characterizing the feature, the most important are:

- factor of accessibility of places controlled,

- factor of facility of defectoscopic examination,

- factor of constructional adaptation,

- factor of defectoscopic ergonomy,

- factor of non-dismantling defectoscopic examination, etc.

\section{The defectability of ndt methods to flaw detection}

The defectability of ndt methods to flaw detection, as a feature of defectoscopic susceptibility, is connected with an application of various nondestructive methods to detection of such discontinuities of machine elements as flaws, cracks and others. To a sufficient quantitative description of this feature the following quantities can be included:

- universality of defectoscopic method,

- likelihood of defectoscopic examination,

- factor of automotion of defectoscopic activity,

- a complex factor of adaptation of nondestructive method to defectoscopic examination, etc.

\section{Non-technical factors of defectoscopic susceptibility}

The following operational quantities belong to this group of factors:

- time of effective defectoscopic examination,

- time of preparation to defectoscopic examination,

- time of removal of possible effects of examination,

- operational factor, etc.

Another group of non-technical quantities comprises such economical factors as [7]:

- labour consumption of defectoscopic examination,

- labour consumption of all bookkeeping operations,

- costs of defectoscopic examination,

- costs of preparation and termination of defectoscopic examination,

- direct and indirect costs of defectoscopic examination,

- material costs and amortization charges of testing equipment,

- generalized factor of cost of defectoscopic examination, etc.

\section{A structure of some factors}

Out of the group of factors characterizing ability of elements to defectoscopic examination we can present the factor of accessibility of places controlled $K_{d m}$ :

$$
K_{d m}=T_{o} / T_{o}+T_{d}
$$

where: $T_{o}$ - labour consumption of basic activity,

$T_{d}$ - labour consumption of bookkeeping operations.

Representing the defectability of ndt methods to flaw detection, a factor of automotion of defectoscopic activity $K_{A}$, can be described by the formula:

$$
K_{A}=\sum_{i=1}^{m} N_{A i} / \sum_{j=1}^{n} N_{O j}
$$


where: $\sum N_{A i}$-a number of parameters automatically measured and worked out,

$\sum N_{O j}$-general number of parameters used.

As an example of non-technical factors, we can use the factor of labour consumption of all bookkeeping operations $K_{p}$ :

$$
K_{p}=1-T_{p} / T_{d}
$$

where: $T_{p}$ - an average labour consumption of all bookkeeping operations,

$T_{d}$ - an average operational labour consumption of defectoscopic examination of an object.

In the same way, factors of other features of machine elements, connections, subassemblies and devices can be mathematically described as, for example, diagnostic or service susceptibility.

\section{Examples of determination of susceptibilities}

For numerical illustration of the problem discussed in the paper, the example of evaluation of defectoscopic susceptibility for selected parts of automotive vehicle is shown in Table 1. This evaluation was calculated on the basis of factors mentioned above. The method of multicriterial analysis was used in this case. The assumed scale of defectoscopic susceptibility was from 1 to 5 , i.e. very low-1, low-2, medium-3, high-4, very high-5, and the assumed factors weight from 1 to 3, i.e. low-1, medium-2, high-3 [4].

The calculated factor of defectoscopic susceptibility of elements $W_{d s}$ was determined with the aid of the following formula:

$$
W_{d s}=\sum_{i=1}^{n}\left(W_{i} O_{i}\right) / \sum_{i=1}^{n} W_{i}
$$

where: $W$ - weight coefficient of each criterion considered,

$\mathrm{O}$ - scale of influence of each criterion on defectoscopic susceptibility,

$n$ - number of criteria considered.

Analyzed parts of automobile vehicles were divided into three groups depending on the required dismantling and the values of their calculated factors of defectoscopic susceptibility are gathered in Table 1.

On the basis of the calculations carried out (Table 1) it can be noticed that out of those parts of automotive vehicle where dismantling is not required, the highest defectoscopic susceptibility was obtained at the brake disc or chassis of a car. Out of those parts where partial dismantling is required, the best susceptibility is at the drive shaft, and out of these parts where full dismantling is required, the brake drum has the highest value.

Nondestructive methods of flaw detection are connected with the diagnosis of machines [8], thus another example of susceptibility evaluation by calculation of diagnostic susceptibility is presented in Table 2. In this example the same assumptions were made and identical calculations were carried out. The same assembly air conditioning of some popular models of cars was analyzed. The analysis of diagnostic susceptibility was carried out separately for subassemblies responsible for the flow of refrigerant (refrigerating medium) and responsible for the functioning of the car air conditioning system control. The weights of factors influencing the diagnostic susceptibility of the system were adapted on the basis of the opinion of the experienced servicemen of these systems [9].

On the basis of the analysis carried out (Table 2), it can be noticed that Toyota Prius has the highest factor of diagnostic susceptibility for air the conditioning system, mainly thanks to a con-

Calculated results of defectoscopic susceptibility factor for selected parts of car

Tab. 1

\begin{tabular}{|c|c|c|c|c|c|}
\hline \multicolumn{6}{|c|}{ Calculated values of defectoscopic susceptibility factor for parts of car } \\
\hline \multicolumn{2}{|c|}{ No dismantling required } & \multicolumn{2}{|c|}{ Partial dismantling required } & \multicolumn{2}{c|}{ Full dismantling required } \\
\hline element & value & element & value & element & value \\
\hline Brake disc & 3.7 & Drive shaft & 3.5 & Brake drum & 3.4 \\
\hline Chassis & 3.5 & Axle casing & 3.2 & Steering knuckle & 3.3 \\
\hline Suspension spring & 3.1 & Stabilizer & 3.1 & Piston & 3.0 \\
\hline Ball joint & 2.6 & Valves & 3.0 & Crank shaft & 2.7 \\
\hline Bolt of road wheel & 2.5 & - & - & Cam shaft & 2.6 \\
\hline
\end{tabular}

Calculated results of diagnostic susceptibility factor of air condition systems for the selected cars

Tab. 2

\begin{tabular}{|l|c|c|c|c|c|c|}
\hline \multirow{2}{*}{ Factor of diagnostic susceptibility } & \multicolumn{6}{|c|}{ Model of car } \\
\cline { 2 - 8 } & $\begin{array}{c}\text { Toyota } \\
\text { Avensis }\end{array}$ & $\begin{array}{c}\text { Toyota } \\
\text { Prius }\end{array}$ & $\begin{array}{c}\text { Hyundai } \\
\text { Accent }\end{array}$ & $\begin{array}{c}\text { Daewoo } \\
\text { Leganza }\end{array}$ & $\begin{array}{c}\text { Opel } \\
\text { Astra III }\end{array}$ & $\begin{array}{c}\text { Fiat } \\
\text { Seicento }\end{array}$ \\
\hline Refrigerant flow devices & 2.4 & 3.0 & 3.7 & 2.9 & 2.8 & 3.8 \\
\hline System control devices & 3.4 & 3.7 & 2.4 & 3.1 & 3.3 & 2.2 \\
\hline Whole conditioning system & 5.8 & 6.7 & 6.1 & 6.0 & 6.1 & 6.0 \\
\hline
\end{tabular}


siderable range and facility of receiving the information from the system of air conditioner automatic control. The access to elements of refrigerant flow is difficult because of compact settlement of engine chamber (hybridized drive, safety equipment, etc.).

Such models as Hyundai Accent and Fiat Seicento also have good diagnostic susceptibility of the conditioning system. In these cases the access to elements of the systems is easy, but considerably less diagnostic information can be derived from programmers of automatic control of the systems. The Toyota Avensis has difficult access to the system considered. The systems of such cars as Opel Astra III and Daewoo Leganza obtained average calculated values of evaluation of their diagnostic the susceptibility.

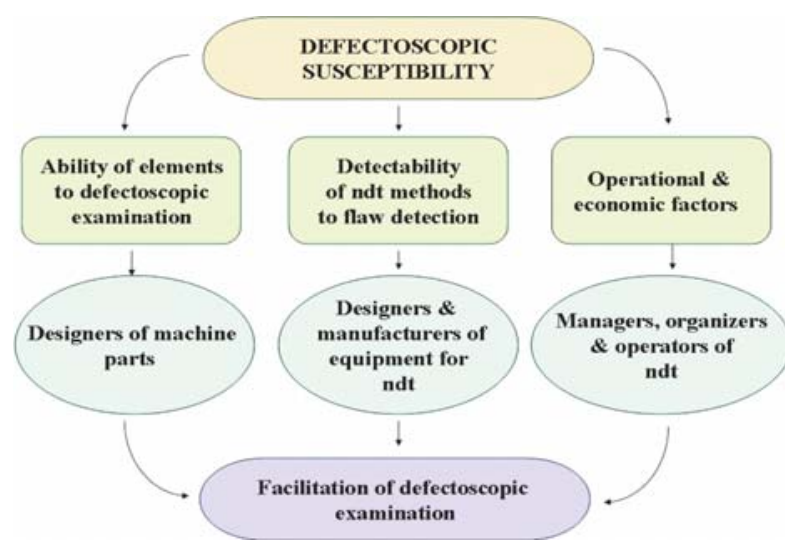

Fig. 3. The scheme of dependencies between defectoscopic susceptibility and simplification of defectoscopic examination

Quantitative information about the values of defectoscopic susceptibility of machine parts or other features such as defectoscopic, diagnostic or service susceptibility can be useful for designers of machine parts and assemblies, organizers or operators of such processes as nondestructive evaluation, diagnosis or service of machines and their subassemblies. Relations illustrating the practical side of the problem discussed in this work are shown in Fig. 3.

\section{Recapitulation}

The defectoscopic susceptibility is a feature, which appears during nondestructive tests, particularly during defectoscopic control.

The defectoscopic susceptibility can be parameterized and considered as the system containing, as a minimum, the subsystem of an ability of machine elements and their connection to nondestructive testing, and the subsystem of detectability of nondestructive testing method to detection of discontinuities in the machine elements.

The defectoscopic susceptibility can be represented by some technical and non-technical set of quantities.

A formation of the defectoscopic susceptibility of elements and their joints occurs throughout all the "life time" of machine elements, but in particular, during construction and production of machine elements.

The defectoscopic susceptibility, apart from describing that feature, helps us to improve the form (shape, dimensions) of the elements to make the detection of discontinuities in elements during defectoscopic examination easier.

The collections of factors allow us quantitatively evaluate the susceptibility of both sides of the problem: the object of examination and the method of examination.

The problem of defectoscopic susceptibility is still developing. It still needs formal precision and complementation for the application to a greater number of machine real parts with the use of a computer multi criteria analysis method of the presented problem.

\section{References}

[1] PAWLOWSKI, Z.: The Tasks for NDT in development and production of machinery, Proceeding of V Conference CPBP, Warsaw, 1992, pp. 87-100.

[2] DON, E. BRAY, RODERIC STANLEY, K.: Nondestructive Evaluation, A Tool in Design, Manufacturing and Service, Mc Graw-Hill, New York, 1989.

[3] WHITTINGTON, H.W., FLYNN, B.W.: High Reliability Condition Monitoring Systems, British Journal of Nondestructive Testing, 1993, Vol. 35, No 11, pp. 648-654.

[4] NOWAK, K.: An analysis of defectoscopic susceptibility of selected element of a car (in Polish), A thesis - unpublished, Poznan University of Technology. 2003, $66 \mathrm{p}$.

[5] ZOLTOWSKI, B.: A Basis of Technical Diagnostics (in Polish), ATR, Bydgoszcz, 1996.

[6] DRECHSLER, J.: A design friendly to examination by nondestructive testing methods (in German), A paper of International Seminar TEMPUS S-JEP-07945, 24. 04. 1997, Institute of Materials Engineering, Technical University of Szczecin.

[7] SOZANSKA, I.: Costs of quality in laboratory of enterprise (in Polish), Problems Books - Nondestructive Testing Methods. 1997, No 2, pp. 161-172.

[8] CWIK, Z.: Determination of diagnostic susceptibility factor of machines (in Polish), Problems of Maintenance, 1997, No 4, pp. 109-118.

[9] CHORAZYCZEWSKI, M.: An Analysis of diagnostic susceptibility of air conditioning system of automotive vehicles (in Polish), A thesis - unpublished, Poznan University of Technology. 2005, 78p. 